\title{
Reaction of 1-aminoisoindole with methyl 4-chloro-3-oxobutanoate
}

\author{
Kysil Andrii", Biitseva Angelina, Yegorova Tetyana, Levkov Igor, Voitenko Zoia \\ Department of Chemistry, Taras Shevchenko National University of Kyiv, Volodymyrska Street, \\ 64/13, Kyiv 01601, Ukraine \\ kysil425@ukr.net
}

Keywords: 1-aminoisoindole, methyl 4-chloro-3-oxobutanoate, pyrimido[2,1-a] isoindol-4(3H)-one, amines, heterocyclization, regioisomers, heteronuclear correlation spectroscopy.

Condensation of bifunctional 1-aminoisoindole with bis-electrophilic methyl 4-chloro-3oxobutanoate undergoes regioselectively to afford 2-(chloromethyl)-2-hydroxy-2,6-dihydropyrimido[2,1-a]isoindol-4(3H)-one. The structure of the reaction product was unambiguously established by HMQC and HMBC heteronuclear correlations. The functionalization of the synthesized compound by reactions with a series of aliphatic amines was carried out.

\section{Introduction}

Isoindole derivatives are widely used for the development of new drugs [1-7], as dyes, pigments and fluorophores in materials science [1,8-11], as well as markers in analytical chemistry [1].

The 1-aminoisoindole is often used as unsymmetrical 1,3-NCN-binucleophilic building block in the synthesis of various isoindole-based fused heterocyclic systems. Thus, its reaction with ethyl 5-acetyl-3,4-dihydropyridine-1(2H)carboxylate leads to regioselective formation of pyrimido[2,1-a]isoindole derivative [12], while condensation of 1-aminoisoindole with phthaloyl chloride [13] or $o$-chloromethylbenzonitrile and further treatment with sodium ethanolate [14] results in isoindolo[2,1-b][2,4]benzodiazepine derivatives. Reactions of 1-aminoisoindole with various $\alpha$-haloketones, $\beta$-diketones and $\beta$-keto esters leading to the formation of $5 H$-imidazo[2,1- $a$ ]isoindole and $6 H$-pyrimido[2,1- $a]$ isoindole derivatives, respectively, have also been studied [1517]. To the best of our knowledge, condensation of 1 aminoisoindole derivatives with 4-halogen-3oxobutanoates were not described to date, although the latter are very common reagents in heterocyclization with various amidine-containing substrates. The molecule of 4-halogen-3oxobutanoate contains several reaction centers and could play a role of 1,3-CCC- as well as 1,2-CCbiselectrophile; that ensures the variability of the reactions and the synthetical diversity of the products obtained. For example, its condensation with different amidines leads to the formation of substituted pyrrolo[2,3- $d$ ]pyrimidines [18], imidazo[1,2-a]pyridines [19], thiazolo[3,2$b][1,2,4]$ triazoles $\quad[20], \quad$ benzo $[d]$ imidazo[2,1$b]$ thiazoles [21], imidazo[1,2-a]pyrazines [22], pyrido[1,2-a]pyrimidines [23] etc. 


\section{Results and discussion}

Herein, we report our studies on the condensation of 1-aminoisoindole hydrobromide with methyl 4-chloro-3-oxobutanoate. The reaction was carried out in dry dioxane in the presence of triethylamine. As a result of heterocyclization, a product was obtained in moderate yield (46\%). This product may have one of two alternative regioisomeric structures $3 \mathbf{a}$ or $\mathbf{3 b}$ (Scheme 1). It should be noted that

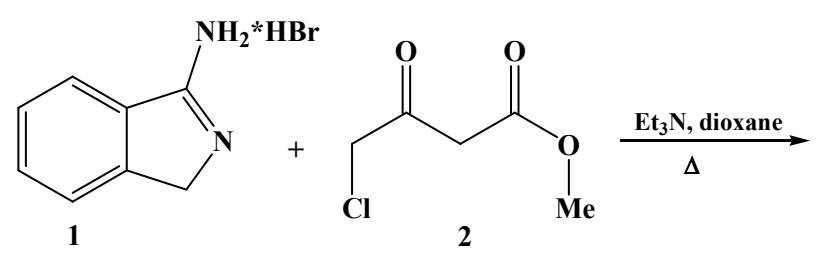

Scheme 1. Reaction of 1-aminoisoindole 1 with methyl-4-chloro-3-oxobutanoate $\mathbf{2}$.

Structures $\mathbf{3 a}$ and $\mathbf{3 b}$ have similar spin systems; therefore, they cannot be distinguished on the basis of ${ }^{1} \mathrm{H}$ and ${ }^{13} \mathrm{C}$ NMR spectra. To solve this problem, we recorded HMQC and HMBC heteronuclear ${ }^{13} \mathrm{C}-{ }^{1} \mathrm{H}$ correlation spectra of the compound 3 (through one and 2-3 chemical bonds, respectively). The coordinates of the cross-peaks found in the HMQC and HMBC spectra are shown in Table 1.

Correlations through one chemical bond in the HMQC spectrum allowed assigning signals from carbon atoms linked to hydrogen, and quaternary carbon atoms were identified by analysis of correlations through 2-3 chemical bonds in the HMBC spectrum. Here, the most important correlation was the one between the methylene group protons of the isoindoline fragment ( $\delta 4.79 \mathrm{ppm})$ and the carbon atom $\mathrm{C}=\mathrm{O}$ group $(\delta 166.0 \mathrm{ppm})$. Such we have not found any literature examples on reaction of amidines with 4-halogeno-3-oxobutanoates, where both chloromethyl fragment and $\mathrm{OH}$ group were simultaneously present in the product structure at the same carbon atom. Such type of reactions commonly occurred under rather severe conditions, often upon heating in polyphosphoric acid [24], and were accompanied by water molecule elimination.
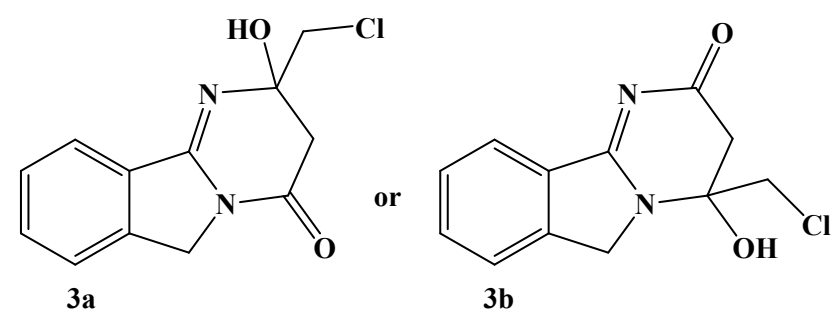

correlation indicates that there are no more than three chemical bonds between these atoms.

Table 1. Heteronuclear ${ }^{1} \mathrm{H}-{ }^{13} \mathrm{C}$ correlations found for compound $\mathbf{3}$.

\begin{tabular}{|c|c|c|}
\hline$\delta, \mathrm{ppm}$ & HMQC & HMBC \\
\hline 7.82 & 124.0 & $155.9 ; 141.2 ; 133.1 ; 124.5$ \\
\hline 7.63 & $133.1 ; 124.5$ & $\begin{array}{c}141.2 ; 135.5 ; 129.0 ; 124.0 ; \\
48.6\end{array}$ \\
\hline 7.47 & 129.0 & $141.2 ; 124.0 ; 124.5$ \\
\hline 6.15 & - & - \\
\hline 4.79 & 48.6 & $\begin{array}{c}166.0 ; 155.9 ; 141.2 ; 135.5 ; \\
129.0 ; 124.5\end{array}$ \\
\hline 3.63 & 52.0 & $86.2 ; 39.0$ \\
\hline 3.68 & 52.0 & $86.2 ; 39.0$ \\
\hline 2.80 & 39.0 & $166.0 ; 86.2 ; 52.0$ \\
\hline 2.62 & 39.0 & $166.0 ; 86.2 ; 52.0$ \\
\hline
\end{tabular}

This allows to assign structure of the reaction product as 2-(chloromethyl)-2-hydroxy-2,6-dihydropyrimido[2,1-a]isoindol-4(3H)-one (3a). In the alternative molecule $\mathbf{3 b}$, the carbonyl carbon atom and the methylene group protons are separated by 5 
chemical bonds, which does not allow for the spin-spin coupling between them.

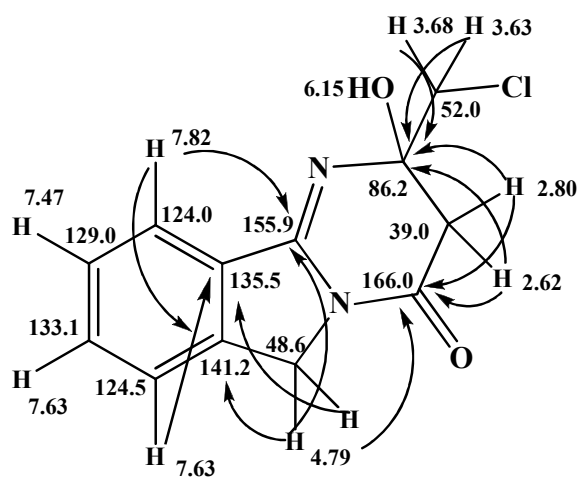

Figure 1. Signals assignment of ${ }^{1} \mathrm{H}$ and ${ }^{13} \mathrm{C}$ NMR spectra for $\mathbf{3 a}$ according to $\mathrm{HMQC}$ and $\mathrm{HMBC}$.
Figure 1 shows the chemical shifts of protons and carbon nuclei in the molecule 3a, as well as the principal correlations in the HMBC spectrum.

To further study chemical properties of pyrimido[2,1-a]isoindolone 3a, we introduced this compound into reactions with a series of aliphatic amines (Scheme 2). The reaction was carried out in dry toluene with large excess of amine, which acted both as a reagent and a base. Derivatives $\mathbf{4}$ were isolated in good yields using column chromatography.

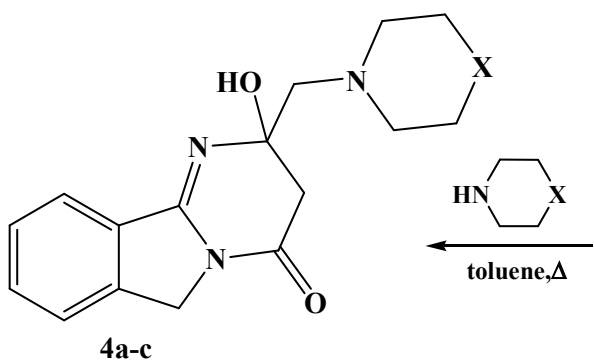

$\mathrm{X}=\mathrm{O}(\mathrm{a}), \mathrm{N}-\mathrm{CH}_{3}(\mathrm{~b}), \mathrm{N}-\mathrm{COH}(\mathrm{c})$

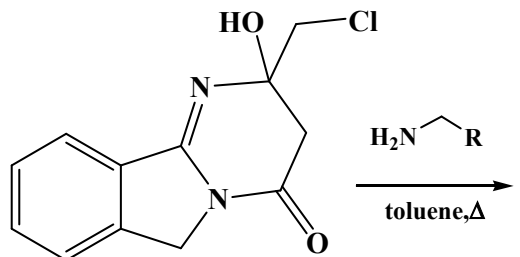

3a

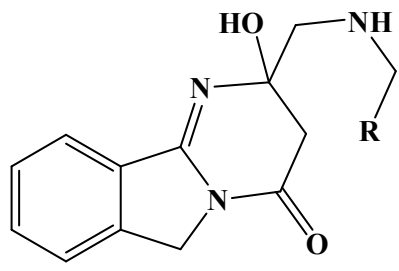

4d-f

$\mathrm{R}=\mathrm{CH}_{2} \mathrm{CH}_{3}(\mathrm{~d}), \mathrm{CH}_{2} \mathrm{CH}_{2} \mathrm{OCH}_{3}(\mathrm{e}), \mathrm{CH}_{2} \mathrm{Ph}$ (f)

Scheme 2. Reaction of $\mathbf{3 a}$ with aliphatic amines.

\section{Experimental part}

NMR spectra were obtained on Mercury 400 Varian spectrometer at $400 \mathrm{MHz}\left({ }^{1} \mathrm{H} \mathrm{NMR}\right)$ or 100 $\mathrm{MHz}\left({ }^{13} \mathrm{C} \mathrm{NMR}\right)$ in DMSO- $d_{6}$. All chemical shifts were referenced to the TMS residual peak as an internal standard. The heteronuclear HMQC and HMBC correlation spectra were recorded using a method of indirect detection of carbon signals and gradient selection of useful signals. Melting points were determined with a Boetius microscope hot plate apparatus. Mass spectra were recorded on an Agilent 1100 LC/MSD SL instrument with chemical ionization.

\section{2-(Chloromethyl)-2-hydroxy-2,6-} dihydropyrimido[2,1-a]isoindol-4(3H)-one (3a).
The mixture of 2-aminoisoindole hydrobromide (0.43 g, 2 mmol), methyl 4-chloro-3oxobutanoate $(0.38 \mathrm{~g}, 2.5 \mathrm{mmol})$ and triethylamine $(0.25 \mathrm{~g}, 2.5 \mathrm{mmol})$ in dry dioxane $(10 \mathrm{ml})$ were heated under reflux for $40 \mathrm{~min}$. Precipitate formed after cooling was filtered off and purified by column chromatography on silica gel with ethyl acetatetoluene (8:2) as eluent.

Pale yellow powder(Yield 0.23 g, $46 \%$ ). M.p. 174-175 ${ }^{\circ} \mathrm{C} .{ }^{1} \mathrm{HNMR}: \delta=2.62(\mathrm{~d}, 1 \mathrm{H}, J=16 \mathrm{~Hz}), 2.80$ (d, 1H, J=16 Hz), 3.63 (d, 1H, J=12 Hz), 3.68 (d, 1H, $J=12 \mathrm{~Hz}), 4.79$ (s, 2H), 6.15 (s, 1H, OH), 7.47 (t, 1H, $J=8 \mathrm{~Hz}) 7.49-7.63(\mathrm{~m}, 2 \mathrm{H}), 7.82(\mathrm{~d}, 1 \mathrm{H}, J=8 \mathrm{~Hz}) \mathrm{ppm}$. ${ }^{13} \mathrm{C}$ NMR: $\delta=39.0,48.6,52.0,86.2,124.0,124.5$, 
$129.0,133.1,135.5,141.2,155.9,166.0 \mathrm{ppm} .[\mathrm{M}+\mathrm{H}]^{+}$ 251.

General procedure for preparation of amino alcohols 4.

The mixture of compound 3a $(0.5 \mathrm{~g}, 2 \mathrm{mmol})$ and aliphatic amine $(3 \mathrm{mmol})$ in dry toluene $(10 \mathrm{ml})$ were heated under reflux for $1 \mathrm{~h}$. The solvent was evaporated and the residue was separated by column chromatography on silica gel with hexane-ethyl acetate (9:1) as eluent.

\section{2-Hydroxy-2-(morpholin-4-ylmethyl)-2,6-} dihydropyrimido[2,1-a]isoindol-4(3H)-one (4a).

White powder (Yield $93 \%$ ). M.p. 192-193 ${ }^{\circ} \mathrm{C} .{ }^{1} \mathrm{H}$ NMR: $\delta=2.50-2.55(\mathrm{~m}, 2 \mathrm{H}), 2.57-2.61(\mathrm{~m}$, $4 \mathrm{H}), 3.47$ (s, 2H), 3.65-3.71 (m, 4H), 5.05 (s, 1H), 6.37 (s, 1H, OH), 7.59 (t, 1H, J=7.4 Hz), 7.67 (t, 1H, $J=8 \mathrm{~Hz}), 7.76-7.78(\mathrm{~d}, 1 \mathrm{H}, J=8 \mathrm{~Hz}), 7.99-8.01$ (d, $1 \mathrm{H}$, $J=8 \mathrm{~Hz}$ ) ppm. ${ }^{13} \mathrm{C}$ NMR $\delta=41.0,49.2,53.5,62.8$, 65.8, 85.1, 125.2, 125.5, 127.1, 134.4, 136.0, 142.1, 155.5, 166.7 ppm. $[\mathrm{M}+\mathrm{H}]^{+} 302$.

\section{2-Hydroxy-2-[(4-methylpiperazin-1-}

yl)methyl]-2,6-dihydropyrimido[2,1-a]isoindol4(3H)-one (4b)

Pale yellow powder (Yield 74 \%). M.p. 187-188 ${ }^{\circ} \mathrm{C} .{ }^{1} \mathrm{H}$ NMR: $\delta=2.44(\mathrm{~s}, 3 \mathrm{H}), 2.51-2.55$ (m, 2H), 3.35-3.40 (m, 4H), $3.51(\mathrm{~s}, 2 \mathrm{H}), 3.95-4.11(\mathrm{~m}$, 4H), 5.15 (s, 2H), 6.42 (s, 1H, OH), 7.61 (t, 1H, J=7.4 Hz), 7.69 (t, 1H, $J=8 \mathrm{~Hz}), 7.72-7.76(\mathrm{~d}, 1 \mathrm{H}, J=8 \mathrm{~Hz})$, 7.96-7.98 (d, $1 \mathrm{H}, J=8 \mathrm{~Hz})$ ppm. ${ }^{13} \mathrm{C} \mathrm{NMR}: \delta=40.2$, 46.0, 48.5, 53.1, 54.5, 64.1, 86.5, 123.8, 125.7, 128.3, 133.9, 136.3, 144.0, 157.1, 165.8 ppm. $[\mathrm{M}+\mathrm{H}]^{+} 315$.
4-[(2-Hydroxy-4-oxo-2,3,4,6-

tetrahydropyrimido[2,1-a]isoindol-2-

yl)methyl]piperazine-1-carbaldehyde (4c)

Pale yellow powder (Yield $76 \%$ ). M.p. 190-192 ${ }^{\circ} \mathrm{C} .{ }^{1} \mathrm{H}$ NMR: $\delta=2.50-2.53(\mathrm{~m}, 2 \mathrm{H})$, $3.32-2.38(\mathrm{~m}, 4 \mathrm{H}), 3.48$ (s, 2H), 3.97-3.99 (m, 2H), 4.02-4.04(m, 2H), $5.18(\mathrm{~s}, 2 \mathrm{H}), 6.40(\mathrm{~s}, 1 \mathrm{H}, \mathrm{OH}), 7.62$ (t, 1H, $J=7.4 \mathrm{~Hz}$ ), 7.65 (t, 1H, $J=8 \mathrm{~Hz}), 7.73-7.75$ (d, $1 \mathrm{H}, J=8 \mathrm{~Hz}$ ), 7.91-7.93 (d, 1H, $J=8 \mathrm{~Hz}), 8.40$ (s, 1H) ppm. ${ }^{13} \mathrm{C}$ NMR $\delta$ (DMSO-d $)$ 38.1; 42.1; 49.1; 51.8; $62.2 ; 87.7 ; 125.2 ; 125.7 ; 129.6 ; 134.1 ; 135.9 ; 141.8$; $156.9 ; 160.1 ; 166.8$ ppm. $[\mathrm{M}+\mathrm{H}]^{+} 329$.

2-Hydroxy-2-[(propylamino)methyl]-2,6-

dihydropyrimido[2,1-a]isoindol-4(3H)-one (4d)

Pale yellow powder (Yield $92 \%$ ). M.p. 162-163 ${ }^{\circ} \mathrm{C} .{ }^{1} \mathrm{H}$ NMR: $\delta=1.04(\mathrm{t}, 3 \mathrm{H}), 1.70-1.79(\mathrm{~m}$, $2 \mathrm{H}), 3.57-3.61(\mathrm{~m}, 2 \mathrm{H}), 3.52(\mathrm{~s}, 2 \mathrm{H}), 5.14(\mathrm{~s}, 2 \mathrm{H})$, 6.34 (s, 1H, OH), 7.55 (t, 1H, J=7.4 Hz), $7.62(\mathrm{t}, 1 \mathrm{H}$, $J=8 \mathrm{~Hz}), 7.70-7.72(\mathrm{~d}, 1 \mathrm{H}, J=8 \mathrm{~Hz}), 7.88-7.90(\mathrm{~d}, 1 \mathrm{H}$, $J=8 \mathrm{~Hz}$ ) ppm. ${ }^{13} \mathrm{C}$ NMR: $\delta=10.8,21.5,39.7,48.2$, 50.1, 59.0, 87.0, 125.3, 125.9, 130.3, 134.3, 137.0, 142.4, 157.1, 167.1 ppm. $[\mathrm{M}+\mathrm{H}]^{+} 274$.

2-Hydroxy-2-\{[(3-

methoxypropyl)amino]methyl $\}-2,6-$

dihydropyrimido[2,1-a]isoindol-4(3H)-one (4e)

White powder (Yield 73 \%). M.p. 167-168 ${ }^{\circ} \mathrm{C} .{ }^{1} \mathrm{H}$ NMR: $\delta=2.54-2.56(\mathrm{~m}, 2 \mathrm{H}), 3.21(\mathrm{~s}, 3 \mathrm{H}$, $\left.\mathrm{OCH}_{3}\right), 3.50-3.52(\mathrm{~m}, 2 \mathrm{H}), 3.96-3.98(\mathrm{~m}, 2 \mathrm{H}), 3.48$ (s, 2H), $5.04(\mathrm{~s}, 2 \mathrm{H}), 6.29(\mathrm{~s}, 1 \mathrm{H} \mathrm{OH}), 7.53(\mathrm{t}, 1 \mathrm{H}$, $J=7.4 \mathrm{~Hz}), 7.65$ (t, 1H, $J=8 \mathrm{~Hz}), 7.69-7.71$ (d, 1H, $J=8$ $\mathrm{Hz}), 7.92-7.94(\mathrm{~d}, 1 \mathrm{H}, J=8 \mathrm{~Hz}) \mathrm{ppm} .{ }^{13} \mathrm{C} \mathrm{NMR}: \delta=$ 31.6, 39.5, 47.4, 49.6, 56.2, 57.2, 67.1, 87.7, 126.1, 
$126.8,130.2,134.2,136.1,143.1,156.7,167.2 \mathrm{ppm}$. $[\mathrm{M}+\mathrm{H}]^{+} 304$.

\section{2-Hydroxy-2-\{[(2-} phenylethyl)amino] methyl\}-2,6-dihydropyrimido[2,1a]isoindol-4(3H)-one (4f)

Pale yellow powder (Yield 92 \%). M.p. 191 ${ }^{\circ} \mathrm{C} .{ }^{1} \mathrm{H}$ NMR: $\delta=2.79-2.84(\mathrm{~m}, 4 \mathrm{H}), 3.45(\mathrm{~s}, 2 \mathrm{H}), 5.03$ (s, 2H), 6.30 (s, 1H, OH), 7.14-7.25 (m, 5H), 7.59 (t, $1 \mathrm{H}, J=7.4 \mathrm{~Hz}), 7.67$ (t, $1 \mathrm{H}, J=8 \mathrm{~Hz}), 7.74-7.76(\mathrm{~d}, 1 \mathrm{H}$, $J=8 \mathrm{~Hz}), 7.95-7.97(\mathrm{~d}, 1 \mathrm{H}, J=8 \mathrm{~Hz}) \mathrm{ppm} .{ }^{13} \mathrm{C} \mathrm{NMR}: \delta$ $=34.5,39.8,48.5,48.9,57.2,87.0,124.6,124.9,125.9$, $126.8,127.5,131.1,134.2,136.7,138.1,143.2,156.4$, 167.7 ppm. $[\mathrm{M}+\mathrm{H}]^{+} 336$.

\section{Conclusions}

Reaction of 1-aminoisoindole with methyl-4chloro-3-oxobutanoate occurs in a regioselective manner to provide 2-(chloromethyl)-2-hydroxy-2,6dihydropyrimido[2,1-a]-isoindol-4-(3H)-one 3a. A simple approach to novel biologically promising amino alcohols 4 was elaborated, which includes reaction of compound 3a with aliphatic amines.

\section{References}

[1] Speck K, Magauer T. The chemistry of isoindole natural products. Beilstein Journal of Organic Chemistry 2013;9:2048-2078.

[2] Bhatia RK. Isoindole Derivatives: Propitious Anticancer Structural Motifs. Current Topics in Medicinal Chemistry2017;17(2):189-207.

[3] O'Donoghue ML, Bhatt D.L, Flather MD, Goto S, Angiolillo DJ, Goodman SG, Zeymer U, Aylward PE, Montalescot G, Ziecina RJ. Atopaxar and its effects on markers of platelet activation and inflammation: results from the LANCELOT
CAD program. Journal of Thrombosis and Thrombolysis 2012;34(1):36-43.

[4] Gao L, Zhao FL., Li SC. Efficacy and Safety of Thrombin-Receptor Antagonist (Atopaxar and Vorapaxar) in Patients with Acute Coronary Syndrome or Coronary Artery Disease-A Meta-Analysis of Randomized Controlled Trials. Value in Health Regional Issues 2015;6:22-32.

[5] LiY, Liu D, Cen S, Proksch P, Lin W. Isoindolinone-type alkaloids from the sponge-derived fungus Stachybotrys chartarum. Tetrahedron 2014;70(39):7010-7015.

[6] Belliotti TR., Brink WA., Kesten SR., Rubin JR, Wustrow DJ, Zoski KT, Whetzel SZ, Corbin AE, Pugsley TA, Heffner TG, Wise LD. Isoindolinone enantiomers having affinity for the dopamine D4 receptor. Bioorganic \& Medicinal Chemistry Letters 1998;8(12):1499-1502.

[7] Wang K, Bao L, Qi Q, Zhao F, Ma K, Pei Y, Liu H. Erinacerins C-L, Isoindolin-1-ones with $\alpha$-Glucosidase Inhibitory Activity from Cultures of the Medicinal Mushroom Hericium erinaceus. Journal of Natural Products 2015, 78(1):146-154.

[8] Voitenko ZV, Yegorova TV, Kysil' AI, Andre C, Wolf JG. New cyanine dyes derived from tetrazolo[5,1a]isoindoles. Tetrahedron. 2004;60(1):199-206.

[9] Voitenko ZV, Kysil' AI, Wolf JG. New meso substituted cyanine dyes in the 2-R-5H-[1,2,4]triazolo[5,1-a] isoindole series. Dyes and Pigments 2007;74(2):477-482.

[10] Rodik RV, Malytskyi VY, Starova VS, Yegorova TV, Kysil AI, Zaporozhets OA, Voitenko ZV, Kalchenko VI. Synthesis, fluorescent properties and aggregation of 2,3dihydroisoindolenylcalix[4]arenes. Journal of Inclusion Phenomena and Macrocyclic Chemistry 2013;77:343-350.

[11] Yu C, Xu Y, Jiao L, Zhou J, Wang Z, Hao E. Isoindole BODIPY Dyes as Red to Near Infrared Fluorophores. Chemistry A European Journal 2012;18(21):6437-6442.

[12] Maximov NB, Mykhailiuk PK, Kisel AI, Voitenko ZV, Tolmachev AA. Reaction of Ethyl 5-Acetyl-3,4dihydropyridine-1 $(2 H)$-carboxylate with 1,3- $N, N$-Bisnucleophiles: A Facile Access to Novel Pyrimidine Derivatives. Synthesis 2011;9:1465-1471.

[13] Lessel J. Benzodiazepines and isoindoles by acylation of amidines. Pharmazie 1993;48(11):812-816. 
[14] Tyltin AK, Lysik NA, Demchenko AM, Kovtunenko VA. Synthesis and reactions of derivatives of isoindolo[2,1-b][2,4]benzodiazepine. Chemistry of Heterocyclic Compounds 1985;21: 598.

[15] Toja E, Omodei-Salé A, Favara D, Cattaneo C, Gallico L, Galliani G. Synthesis and pregnancy terminating activity of 2-arylimidazo[2,1-a]isoquinolines and isoindoles. Arzneimittel-Forschung 1983;33(9):1222-1225.

[16] Gruppo Lepetit, s.p.A. - US4075342A, 1978. Antireproductive imidazo[2,1-a]isoquinoline compounds.

[17] Tyltin AK, Kovtunenko VA, Babichev FS. 6-Hpyrimido[2,1-a]isoindolium perchlorates. Chemistry of Heterocyclic Compounds 1979;15:812.

[18] Xing R, Zhang H, Yuan J, Zhang K, Li L, Guo H, Zhao L, Zhang C, Li S, Gao T, Liu Y, Wang L. Novel 6-substituted benzoyl and non-benzoyl straight chain pyrrolo[2,3- $d$ ]pyrimidines as potential antitumor agents with multitargeted inhibition of TS, GARFTase and AICARFTase. European Journal of Medicinal Chemistry 2017;139:531-541.

[19] N'Guessan, JDU, Delaye P, Pénichon M, Charvet CL, Neveu C, Ouattara M, Enguehard-Gueiffier C, Gueiffier A, Allouchi H. Discovery of imidazo[1,2-a]pyridine-based anthelmintic targeting cholinergic receptors of Haemonchus contortus. Bioorganic and Medicinal Chemistry 2017;25(24):6695-6706.

[20] Toma A, Mogoşan C, Vlase L, Leonte D, Zaharia V, Heterocycles 39. Synthesis, characterization and evaluation of the anti-inflammatory activity of thiazolo[3,2- $b][1,2,4]$ triazole derivatives bearing pyridin-3/4-yl moiety. Medicinal Chemistry Research 2012;26(10):2602-2613.

[21] Roslan II, Ng KH, Chuah GK, Jaenicke S. Reagentcontrolled regiodivergent intermolecular cyclization of 2aminobenzothiazoles with $\beta$-ketoesters and $\beta$-ketoamides. Beilstein Journal of Organic Chemistry 2017;13:2739-2750.

[22] Ratni H, Karp GM, Weetall M, Naryshkin NA, Paushkin SV, Chen KS, McCarthy KD, Qi H, Turpoff A, Woll MG, Zhang X, Zhang N, Yang T, Dakka A, Vazirani P, Zhao X, Pinard E, Green L, David-Pierson P, Tuerck D, Poirier A, Muster W, Kirchner S, Mueller L, Gerlach I, Metzger F. Specific Correction of Alternative Survival Motor Neuron 2 Splicing by
Small Molecules: Discovery of a Potential Novel Medicine to Treat Spinal Muscular Atrophy. Journal of Medicinal Chemistry 2016;59(13):6086-6100.

[23] Villemure E, Volgraf M, JiangY, Wu G, Ly CQ, Yuen PW, Lu A, Luo X, Liu M, Zhang S, Lupardus PJ, Wallweber HJA, Liederer BM, Deshmukh G, Plise E, Tay S, Wang TM, Hanson JE, Hackos DH, Scearce-Levie K, Schwarz JB, Sellers BD. GluN2A-selective pyridopyrimidinone series of nmdar positive allosteric modulators with an improved in vivo profile. ACS Medicinal Chemistry Letters 2016;8(1):84-89.

[24] Volgraf M, Sellers BD, Jiang Y, Wu G, Ly CQ, Villemure E, Pastor RM, Yuen P, Lu A, Luo X, Liu M, Zhang S, Sun L, Fu Y, Lupardus PJ, Wallweber HJA, Liederer BM, Deshmukh G, Plise E, Tay S, Reynen P, Herrington J, Gustafson A, Liu Y, Dirksen A, Dietz MGA, Liu Y, Wang TM, Hanson JE, Hackos D, Scearce-Levie K, Schwarz JB. Discovery of GluN2ASelective NMDA Receptor Positive Allosteric Modulators (PAMs): Tuning Deactivation Kinetics via Structure-Based Design. Journal of Medicinal Chemistry 2016;59(6):2760-2779. 